\title{
Calidad microbiológica de cilantro (Coriandrum sativum L.) para la venta al público
}

\author{
Coriander's microbiology quality (Coriandrum sativum L.) \\ for sale to public
}

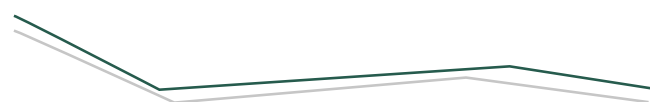

Marisol Santos Fernández*, Valeria Maldonado Ortega*, Yisa Ma. Ochoa Fuentes*®, Ernesto Cerna Chávez*, Omegar Hernández Bautista**

Santos Fernández, M., Maldonado Ortega, V., Ochoa Fuentes, Y. M., Cerna Chávez, E., \& Hernández Bautista, O. (2018). Calidad microbiológica de cilantro (Coriandrum sativum L.) para la venta al público. Investigación y Ciencia de la Universidad Autónoma de Aguascalientes, 26(74), 5-9.
\end{abstract}

\section{RESUMEN}

Se determinó la calidad microbiológica del cilantro (Coriandrum sativum L.) de diversos centros comerciales de Saltillo, Coahuila, México, a través de la determinación de índices microbiológicos. Se empleó la metodología descrita en la norma NOM112-SSA 1-1994 para la determinación de coliformes totales por el NMP, la determinación de parásitos se hizo por concentración y observación directa. Como resultado se obtuvo que los supermercados analizados están fuera de los límites microbiológicos, tanto para coliformes totales como parásitos, siendo Ascaris lumbricoides el de mayor presencia. Las muestras de cilantro rebasaron los límites permisibles que marca la norma, posiblemente por carencia en la aplicación de SRRC-SENASICA (México) durante la manipulación previa, así como en la venta.

Palabras clave: cilantro; coliformes; Ascaris lumbricoides; SRRC; calidad microbiológica.

Keywords: coriander; coliforms; Ascaris lumbricoides; SRRC; microbiological quality.

\section{Recibido: 6 de octubre de 2017, aceptado: 20 de marzo de 2018}

* Departamento de Parasitología Agrícola, Universidad Autónoma Agraria Antonio Narro. Calzada Antonio Narro 1923, Col. Buenavista, C. P. 25315 , Saltillo, Coahuila, México. Correo electrónico: msantos.fdz@gmail.com; valeriamaldonado7@hotmail.com; yisa8a@yahoo.com; jabaly1@yahoo. com ** Culta, S. A. de C. V. Blvd. Luis Echeverría Álvarez No. 1700, Col. Altavista,
C. P. 89880 , Cd. Mante, Tamaulipas, México. Correo electrónico: omegarhbautista@gmail.com

$凶$ Autor para correspondencia

\section{ABSTRAC1}

The microbiological quality of the coriander (Coriandrum sativum L.) of several commercial centers of Saltillo, Coahuila, Mexico, was determinated by the methodology described in standard NOM-112-SSA1-1994 for determination of total coliforms and the determination of parasites was done by concentration and direct observation. As a result, all supermarkets analyzed are outside the microbiological limits for total coliforms and parasites, with Ascaris lumbricoides being the most prevalent. Coriander samples were unsatisfactory at quality levels possibly due to lack of application of SRRC-SENASICA (Mexico) during previous handling, as well as in the market.

\section{INTRODUCCIÓN}

El cilantro (Coriandrum sativum L.) es una planta aromática, usualmente se consume fresco y es fuente de vitamina K (Lo Cantore, lacobellis, De Marco, Capasso, \& Senatore, 2004). En países de América Latina habitualmente se ingiere cruda y por el tipo de cultivo y prácticas de consumo puede presentar un gran número de bacterias entéricas y parásitos que son la causa principal de las enfermedades transmitidas por alimentos (ETA) (Cerón Salgado \& Grijalva, 2015). Las ETA representan un peligro para la salud, ya que se producen por el consumo de alimentos contaminados por patógenos y las toxinas que los mismos producen (Ramírez Mérida, Morón de Salim, Alfieri Graterol, \& Gamboa, 2009). 
IIVESTIGACIÓn Y CIERCIA DE LA UNIVERSIDAD AUTÓNOMA DE AGUASCALIERTES
La Organización Mundial de la Salud (OMS, 2015) en 2010 reportó 600 millones de personas enfermas y 420, 000 muertes por consumir alimentos insalubres.

Los microorganismos aislados causantes de las ETA en productos frescos incluyen bacterias (como Escherichia coli, Salmonella spp. y Shigella sp.), protozoarios (Giardia lamblia y amebas las más comunes), helmintos (Ascaris lumbricoides y Taenia sp.) y virus (hepatitis A, rotavirus y virus Norwalk) causantes de infecciones gastrointestinales (AriasEchandi \& Antillón, 2000; Hernández Cortez, Aguilera Arreola, Castro, \& Escarpulli, 2011). En México, de acuerdo con las cifras expedidas por la Dirección General de Epidemiologia (DGE), en 2016 la ETA con mayor incidencia fue la amibiasis, con 155,674 casos reportados; sin embargo, en las cifras emitidas en el reporte de la semana 35 por la misma institución hubo un aumento, llegando a 157,964 en la primera parte del año 2017. También reportan un aumento de todas las demás ETA (con excepción de la helmintiasis, la cual tiene aun cifras menores a la del año anterior) como las producidas por protozoarios, que están reportadas en 53,372 casos, 10,000 más que en 2016 (Secretaría de Salud, 2017).

Debido a la importancia e incremento de las ETA año con año en México y la utilidad de los microorganismos indicadores para evaluar la calidad microbiológica de los alimentos, el presente trabajo tuvo como objetivo determinar la cantidad de coliformes totales y la presencia de parásitos intestinales del cilantro de diversos centros comerciales de Saltillo, Coahuila, México.

\section{MATERIALES Y MÉTODOS}

Esta investigación fue un estudio descriptivo, transversal, con selección de la muestra no probabilística y al azar en los supermercados de la ciudad de Saltillo, Coahuila, México. Se tomaron dos supermercados de cadena internacional (A y $B$ ), dos supermercados de cadena nacional (C y D) y dos supermercados locales (E y F) y se realizaron dos muestreos con tres repeticiones, con diferencia de tres semanas entre muestreos. Las muestras fueron recolectadas en las condiciones usuales de manipulación y venta al público.

Para la determinación de formas infectantes parasitarias se utilizó la metodología modificada de Traviezo-Valles et al. (2013). Del cilantro recolectado se pesaron $40 \mathrm{~g}$ y fueron colocados en un frasco con $800 \mathrm{ml}$ de agua potable envasada en botellones, previamente hervida durante $1 \mathrm{~h}$ para destruir cualquier posible contaminante biológico, el frasco se mezcló por inversión al menos cuatro veces y se dejó en reposo $24 \mathrm{~h}$. Transcurrido el tiempo se retiraron las ramas de cilantro utilizando pinzas estériles y evitando el reflujo del agua, de modo posterior a esto se dejó el agua nuevamente en reposo por $1 \mathrm{~h}$ y se decantaron aproximadamente nueve partes del agua con la ayuda de mangueras estériles de goma, para evitar el reflujo. El sedimento del frasco fue resuspendido y distribuido en cuatro tubos cónicos de $50 \mathrm{ml}$ para ser centrifugado por 5 min a 3000 r.p.m. Finalmente, el sedimento fue montado con Lugol para su observación directa.

La cuantificación de coliformes totales se hizo conforme a lo descrito en la Norma Oficial Mexicana NOM-1 12-SSA 1-1994 (SSA, 1995) mediante la técnica del número más probable. Se tomaron $25 \mathrm{~g}$ de cilantro de cada una de las muestras y fueron colocados en $225 \mathrm{ml}$ de agua peptonada, se realizaron las diluciones seriadas, de las cuales se tomó $1 \mathrm{ml}$, fue depositado en tubos con caldo de lauril sulfato de sodio con la campana de Durham previamente colocada y se incubaron $48 \mathrm{~h}$ a $37^{\circ} \mathrm{C}$. Transcurrido el tiempo de los tubos que presentaron crecimiento y formación de gas, fueron resembrados en caldo bilis verde brillante para la confirmación de coliformes totales e incubados por un periodo de $48 \mathrm{~h} \mathrm{a} 37^{\circ} \mathrm{C}$.

Una vez consignados los datos se le realizó una prueba de normalidad mediante Shapiro-Wilk, los valores de NMP/g se compararon con una prueba de medianas para cada muestreo, se realizó una prueba de Kruskal-Wallis con una insignificancia de $5 \%$, considerando ambos muestreos.

RESULTADOS

El análisis de coliformes totales determinó que 100\% de las muestras procesadas están contaminadas. La tabla 1 muestra los resultados obtenidos del conteo de coliformes totales en cada uno de los supermercados.

Con base en la prueba Shapiro-Wilk ( $W=0.69802$, p-value $=2.634 \mathrm{e}-07$ ) no cumple con los supuestos de normalidad, por lo que se contrastan las medianas; para el primer muestreo el supermercado $C$ de 


\section{InVESTIGACIÓn Y CIERCIR DE LA UחIVERSIDAD AUTÓNOMA DE AGUASCALIERTES}

comercio nacional presentó los valores más bajos, seguido del establecimiento $\mathrm{E}$ (local); por otra parte, el supermercado $B$, perteneciente a una cadena internacional, fue la de mayor riesgo de contaminantes en ambos muestreos. Considerando ambos muestreos, solo se determinó diferencia estadística entre las empresas internacionales A y B, siendo las de menor y mayor NMP/g de coliformes totales, respectivamente.

Se observaron 10 formas infectantes de protozoarios y helmintos, siendo Ascaris lumbricoides el de mayor frecuencia. La tabla 2 enlista las especies parasitarias identificadas y la cantidad de formas infectantes encontradas por muestra de cada supermercado.

\section{DISCUSIÓN}

Como se puede apreciar en la tabla 1, ninguno de los supermercados está dentro de los límites permitidos por SRRC (sistema de reducción de riesgos de contaminación en la producción primaria de vegetales) para coliformes totales (figura 1), en donde se señala como límite permisible cero coliformes fecales y máximo dos NMP/g coliformes totales (SENASICA, 2015), siendo los supermercados locales los de mayor riesgo para la salud. De estos resultados, cuatro coinciden con los intervalos obtenidos por Ginestre Pérez et al. (2009), quienes señalan que $96 \%$ de las muestras de cilantro analizadas fueron positivas entre 104-109 NMP/g

Tabla 1

Comparaciones de NMP/g de coliformes totales en cilantro (Coriandrum sativum L.) en Saltillo, Coahuila, México

\begin{tabular}{|c|c|c|c|c|c|c|c|}
\hline \multirow[b]{2}{*}{ Supermercado } & \multicolumn{3}{|c|}{ Muestreo 1} & \multicolumn{2}{|c|}{ Muestreo 2} & \multirow{2}{*}{$\frac{\text { M. R.** }}{\text { R.IQ. }^{* *}(25-}$} & \\
\hline & r & mediana & R.IQ. $(25-75)^{*}$ & $r$ & mediana & & \\
\hline$A$ & 3 & 225 & $(225-250)^{c}$ & 3 & 1075 & $\begin{array}{l}(1075- \\
1075)^{b}\end{array}$ & $13^{B}$ \\
\hline B & 3 & 600 & $(5625-6000)^{a}$ & 3 & 27500 & $\begin{array}{l}(19500- \\
27500)^{a}\end{array}$ & $26^{\mathrm{A}}$ \\
\hline C & 3 & 3 & $(3-3)^{e}$ & 3 & 11500 & $\begin{array}{l}(11500- \\
19500)^{a}\end{array}$ & $14^{\mathrm{AB}}$ \\
\hline $\mathrm{D}$ & 3 & 525 & $(512-612)^{b}$ & 3 & 5250 & $\begin{array}{l}(2925- \\
5250)^{\mathrm{b}}\end{array}$ & $16^{A B}$ \\
\hline$E$ & 3 & 100 & $(100-100)^{d}$ & 3 & 27500 & $\begin{array}{l}(27500- \\
27750)^{a}\end{array}$ & $18^{A B}$ \\
\hline $\mathrm{F}$ & 3 & 500 & $(425-512)^{\mathrm{b}}$ & 3 & 27500 & $\begin{array}{l}(27500- \\
27750)^{a}\end{array}$ & $22^{A B}$ \\
\hline
\end{tabular}

Nota: r: repeticiones, R. IQ.: Rango intercuantil, M.R.: Media de rangos, *: columna con diferente letra estadísticamente significativa, ${ }^{* *}$ : Columna con su respectivo centro comercial con diferente letra estadísticamente significativa.

Tabla 2

Especies parasitarias identificadas en cilantro (Coriandrum sativum L.) en el municipio de Saltillo, Coahuila

\begin{tabular}{lllllll}
\hline \multirow{2}{*}{ Parásito } & \multicolumn{7}{c}{ Supermercado } & \\
& A & B & C & D & E & F \\
\hline Ascaris lumbricoides & 3 & 0 & 10 & 24 & 7 & 11 \\
Taenia sp & 0 & 0 & 0 & 0 & 0 & 2 \\
Uncinarias sp & 0 & 0 & 0 & 0 & 1 & 0 \\
Entamoeba histolytica/dispar & 0 & 0 & 8 & 2 & 7 & 5 \\
Blastocystis hominis & 1 & 0 & 0 & 2 & 1 & 0 \\
Entamoeba coli & 7 & 3 & 2 & 0 & 0 & 3 \\
Endolimax nana & 0 & 0 & 0 & 0 & 26 & 0 \\
\hline
\end{tabular}

Nota: Elaboración propia. 
8

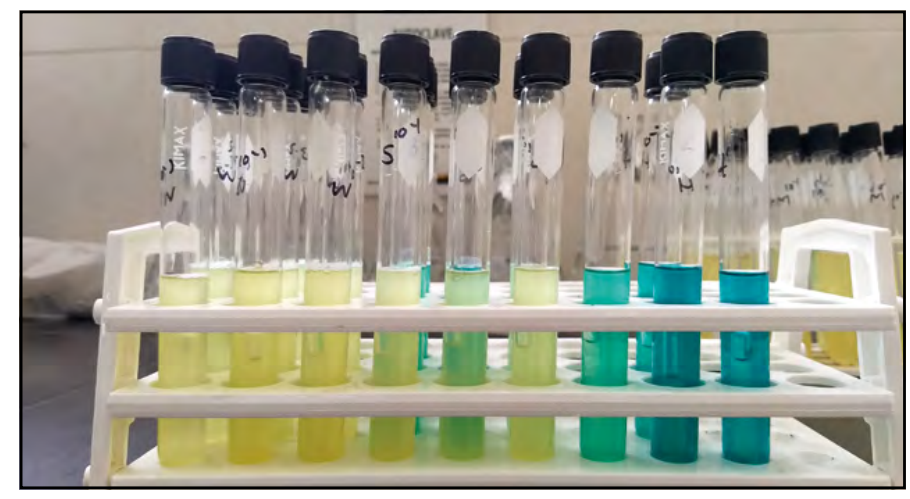

Figura 1. Coliformes totales.

Fotografía del equipo de investigación.

para coliformes totales; asimismo, los resultados coinciden con lo reportado por Noguera Machado et al. (2016), quienes indican que el promedio de coliformes en muestras de cilantro es de $1.1 \times 104$ $\mathrm{NMP} / \mathrm{g}$.

En el análisis parasitológico Ascaris lumbricoides fue el de mayor frecuencia (tabla 2), nematodo intestinal de distribución mundial y de los más comunes que afectan al hombre; se transmite por la ingestión de huevos fecundados, las larvas para desarrollarse pasan a través del pulmón para ser deglutidas y llegar nuevamente al intestino, donde logran llevar su madurez hasta la fase adulta (Vásquez Tsuji et al., 2000). Este helminto se ha logrado identificar en muestras de cilantro en otros estudios realizados con un porcentaje de incidencia entre 5 y $10 \%$ de todas las muestras (Scorza \& Vilchez, 2007; Vilchez \& Scorza, 2007); esta variación puede deberse a que, en México, la ascariasis es la segunda infección gastrointestinal más común (Secretaría de Salud, 2017).
Por otra parte, el resto de los parásitos identificados han sido reportados en porcentajes variados que van desde 0 hasta $40 \%$ (Fallah, PiraliKheirabadi, Shirvani, \& Saei-Dehkordi, 2012; Haq, Maqbool, Khan, Yasmin, \& Sultana, 2014; Hassan, Ojuromi, \& Onyeahialam, 2013). Un parásito que es importante resaltar es Entamoeba coli, un protozoario no patógeno intestinal humano, debido a que solamente vive en el intestino humano; la presencia de $E$. coli en las verduras es el signo de contaminación con heces humanas, por lo que es probable la existencia de organismos intestinales patógenos en dichas verduras (Daryani, Ettehad, Sharif, Ghorbani, \& Ziaei, 2008).

CONCLUSIONES

Los resultados muestran de forma general el estado sanitario de los vegetales y otros productos alimenticios que se expenden y se consumen con alta frecuencia. Los sitios de muestreo presentaron mínima variación para el recuento de coliformes; sin embargo, se encontraron diferencias en la presencia de formas infectantes parasíticas.

Debido a lo observado se pudo clasificar a la mayoría de las muestras de cilantro como insatisfactorias en la calidad que ofrecen al consumidor. La presencia de coliformes totales y parásitos intestinales indica la falta de buenas prácticas de higiene durante el manejo del producto, así como durante su venta. Es recomendable establecer normas de regulación local del estado sanitario que aseguren la inocuidad de este tipo de alimentos considerados de consumo masivo, así como fomentar la capacitación en lo referente a buenas prácticas agrícolas y a buenas prácticas de manejo (SENASICA, 2015). 
- Arias-Echandi, M. L., \& Antillón, G. F. (2000). Contaminación microbiológica de los alimentos en Costa Rica. Una revisión de 10 años. Revista Biomédica, 11 (2), 113-122.

- Cerón Salgado, D., \& Grijalva, N. (2015). Diagnóstico de indicadores entéricos en cilantro (Coriandrum sativum) y perejil (Petroselinum sativum) que se expenden en mercados populares del norte de la ciudad de Quito. Enfoque UTE, 6(1), 45-54.

- Daryani, A., Ettehad, G. H., Sharif, M., Ghorbani, L., \& Ziaei, $H$. (2008). Prevalence of intestinal parasites in vegetables consumed in Ardabil, Iran. Food control, 19(8), 790-794.

- Fallah, A. A., Pirali-Kheirabadi, K., Shirvani, F., \& Saei-Dehkordi, S. S. (2012). Prevalence of parasitic contamination in vegetables used for raw consumption in Shahrekord, Iran: influence of season and washing procedure. Food Control, 25(2), 617-620.

- Ginestre Pérez, M., Romero Añez, S., Rincón Villalobos, G., Castellano González, M., Ávila, R., Colina López, G., \& Perozo Mena, A. (2009). Indicadores entéricos en vegetales frescos que se comercializan en mercados populares de Maracaibo. Revista de la Sociedad Venezolana de Microbiología, 29(1), 52-56.

- Haq, S., Maqbool, A., Khan, U. J., Yasmin, G., \& Sultana, R. (2014). Parasitic contamination of vegetables eaten raw in Lahore. Pakistan Journal of Zoology, 46(5), 1303-1309.

- Hassan, A. A., Ojuromi, O. T., \& Onyeahialam, O. (2013). Presence of parasitic ova, cysts and larva on common fresh fruits and vegetables sold at some major markets in Ibadan, Oyo State, Nigeria. The Zoologist, 11, 40-45.

- Hernández Cortez, C., Aguilera Arreola, M. G., \& Castro Escarpulli, G. (2011). Situación de las enfermedades gastrointestinales en México. Enfermedades infecciosas y microbiología, 31 (4), 137-151.

- Lo Cantore, P., lacobellis, N. S., De Marco, A., Capasso, F., \& Senatore, F. (2004). Antibacterial activity of Coriandrum sativum L. and Foeniculum vulgare Miller var. vulgare (Miller) essential oils. Journal of Agricultural and Food Chemistry, $52(26), 7862-7866$.

- Noguera Machado, N., Ojeda Ojeda, L., Mejía, R., Martínez, F., González, D., \& Requena, D. (2016). Microbiological and parasitological quality of lettuce (Lactuca sativa) and coriander (Coriandrum sativum) expended in the Santa Rita Parish, Aragua, Venezuela. Revista Venezolana de Ciencia y Tecnología de Alimentos, 7(1), 52-64.
Organización Mundial de la Salud. (2015). Estimaciones de la OMS sobre la cara mundial de enfermedades de transmisión alimentaria [Documento en pdf]. Recuperado de http:// apps.who.int/iris/bitstream/10665/200047/1/WHO_FOS_15.02_ spa.pdf

- Ramírez Mérida, L. G., Morón de Salim, A., Alfieri Graterol, A. Y., \& Gamboa, O. (2009). Frecuencia de Listeria monocytogenes en muestras de tomates (Lycopersicum esculentum) y cilantro (Coriandrum sativum) frescos en tres supermercados de Valencia, Venezuela. Archivos Latinoamericanos de Nutrición, 59(3), 318-324.

- Scorza, J. V., \& Vilchez, M. (2007). Recuperación de huevos de Ascaris lumbricoides del follaje de hortalizas intencionalmente contaminadas. Boletín de Malariología y Salud Ambiental, 47(2), 191-194.

- Secretaría de Salud. (1995). NOM-112-SSA1-1994. Bienes y Servicios. Determinación de bacterias coliformes. Técnica del número más probable. Diario Oficial de la Federación, 19 de octubre de 1995.

- Secretaría de Salud. (2017). Boletín epidemiológico. Sistema Nacional de Vigilancia Epidemiológica Sistema Único de Información, 34(39). Recuperado de https://www.gob. $\mathrm{mx} / \mathrm{salud} /$ acciones-y-programas/direccion-general-deepidemiologia-boletin-epidemiologico

- Servicio Nacional de Sanidad, Inocuidad y Calidad Agroalimentaria. (2015). Sistemas de reducción de riesgos de contaminación (SRRC) [Página explicativa]. Recuperada de https://www.gob.mx/senasica/documentos/sistemas-dereduccion-de-riesgos-de-contaminacion-srrc

- Traviezo-Valles, L. E., Salas, A., Lozada, C., Cárdenas, E., Martín, J., \& Agobian, G. (2013). Detección de enteroparásitos en lechugas que se comercializan en el estado Lara, Venezuela. Revista Médico-Científica «Luz Vida», 4(1), 7-11.

- Vásquez Tsuji, O., Gutiérrez Castrellón, P., Yamazaki Nakashimada, M. A., Arredondo Suárez, J. C., Campos Rivera, T., \& Martínez Barbosa, I. (2000). Antihelmínticos como factor de riesgo en la obstrucción intestinal por Ascaris lumbricoides en niños. Boletín chileno de parasitología, 55(1-2), 3-7.

- Vilchez, M., \& Scorza, J. V. (2007). Calidad sanitaria parasitológica de hortalizas cultivadas en La Puerta, municipio Valera, estado Trujillo, Venezuela. Boletín de Malariología y Salud Ambiental, 47(2), 56-59. 